\title{
Ten-year survival with Heart Mate-II in situs viscerum inversus.
}

\author{
Alessandra Francica ${ }^{1}$, Francesco Onorati ${ }^{1}$, Livio San Biagio ${ }^{1}$, Leonardo Gottin ${ }^{1}$, and \\ Giuseppe Faggian ${ }^{1}$ \\ ${ }^{1}$ Integrated University Hospital of Verona
}

June 15, 2020

\begin{abstract}
End-stage heart failure (ESHF) represents the leading cause of death for adult congenital heart disease (ACHD) and heart transplant (HT) remains the first-line treatment. Although many patients are ineligible for transplantation due to advanced comorbidities, left ventricle assist devices (LVAD) is considered a valid option. Herein we present a case of dextrocardia in situs viscerum inversus totalis (SVIT) who was implanted with LVAD HeartMate II ten years ago. To the best of our knowledge, this was the first case of dextrocardia treated with a totally intrathoracic device and he is to date the longest survivor with a HeartMate II in SVIT.
\end{abstract}

\section{Case report}

A 58-year-old man with ischemic cardiomyopathy in SVIT underwent LVAD (HeartMate II; Thoratec Corporation, Pleasanton, CA) implant on November 2009. The postoperative course was uncomplicated and he was discharged home in healthy condition.

In the following years he remained in stable clinical conditions (NYHA class I-II) with a good quality of life $(\mathrm{KCCQ}=79)$. LVAD parameters were always stable. He was regularly followed-up at our Centre (Figure 1). Up to ten years after surgery, chest X-ray never reported signs of pulmonary congestion (Figure 2).

No events of LVAD thrombosis or device dysfunction occurred ever. During time he developed a progressive chronic renal failure with preserved urinary output, now in stage IIIb KDOQI (eGFR $40 \mathrm{ml} / \mathrm{min}$ ). During the last year, the patient needed a new hospitalization for recurrent severe gastrointestinal bleeding, requiring multiple endoscopic treatments, prolonged discontinuation of antiplatelet and anticoagulant regimens (15days during which the INR was always [?]1.4), and transitory administration of octeotride and fresh frozen plasma. Despite that, no thrombotic event occurred, and no LVAD dysfunction developed. Subsequent postoperative course complicated further with pneumonia, successfully treated with antibiotic therapy and non-invasive positive-pressure ventilation. The patient successfully recovered and is next to be discharged in rehabilitation after 4 months of hospitalization.

Last echocardiography (after 123 months from LVAD implant) showed normal inflow and outflow dopplersignals, mild aortic valve regurgitation, and a moderate right ventricular dysfunction with mild tricuspid regurgitation. Despite that, the patient was hemodynamically stable in NYHA class II, with stable LVAD parameters.

Because of the overall successful postoperative course and the good hemodynamic stability, HT was not offered during years.

\section{Comment}

ESHF in patient with CHD who survive into adulthood remains today a challenge for both HT and VAD implantation, because of the complex pathophysiology and potential comorbidities. 
To date, publications regarding outcomes and use of LVADs in ACHD patients are limited.

Of the 16,182 adult patients in the Interagency Registry for Mechanically Assisted Circulatory Support (INTERMACS) database, only $126(0.8 \%)$ have CHD. ${ }^{1}$ Additional analysis on outcomes shows that ACHD patients with LVADs have similar survival, adverse event rates and similar improvements in functional capacity and quality of life compared with non-ACHD patients. ${ }^{6}$ Actually, INTERMACS registry ${ }^{1}$ shows an overall-survival about $70 \%$ at 24 months after implantation of LVADs in ACHD patients, but no data are available on survival beyond 24 post-operative months.

Other literature data on VAD-surgery in ACHD patients consist mainly of case-reports or small series. Of note, besides our patient who reported the first implant ever of a HeartMate II in SVIT ${ }^{2}$, other HeartMate II implantations in adults with SVIT are only reported twice: Ariyachaipanich et al. ${ }^{3}$ described a case of right ventricular support with HeartMate II in a 56-year-old man with a history of SVIT as destination therapy (DT). The patient had an uncomplicated postoperative course and did not require any readmission during the first year after surgery. No data were available beyond the first postoperative year. Menachem et al. ${ }^{4}$ reported a case of a 75-year-old female with SVIT implanted with HeartMate II as DT. She expired early because of neurologic complications.

To the best of our knowledge therefore, this is the first documented case of 10-year survival after HeartMate II implantation in a patient with SVIT. Interestingly enough, long-term follow-up was quite uneventful for more than 8 years, and no device failure, pump thrombosis, or stroke occurred ever during ten-year follow-up, allowing an overall excellent quality of life for a decade. Of note, the prolonged withdrawal of both antiplatelet and anti-thrombotic regimens during the last haemorrhagic event did not led to VAD thrombosis or device malfunction, nor to thromboembolic events, possibly in light of a long-lasting complete formation of neo-intimal tissue with endothelialization of the inner surfaces of the device.

In conclusion, ESHF remains nowadays the leading cause of death for ACHD patients. The ease availability of LVADs provides an elegant solution to these complex scenarios, sometimes allowing long-lasting and consistent results as in the case reported. The recent availability of new generations of these devices can possibly raise the rod further in the next future.

\section{Bibliography}

1. VanderPluym CJ et al. Outcomes following implantation of mechanical circulatory support in adults with congenital heart disease: An analysis of the Interagency Registry for Mechanically Assisted Circulatory Support (INTERMACS). J Hear Lung Transplant. 2018. 2. Faggian G et al. LVAD in situs viscerum inversus totalis. J Hear Lung Transplant, Vol 30, No 12, December 2011 3. Ariyachaipanich A, Mudd JO, Gelow J, Song HK. Long-term systemic right ventricular support in transposition and dextrocardia. J Thorac Cardiovasc Surg. 2012 3. Menachem JN et al. Initial Experience of Left Ventricular Assist Device Support for Adult Patients with Transposition of the Great Vessels. Congenit Heart Dis. 2015 5.Cedars A et al. An Interagency Registry for Mechanically Assisted Circulatory Support (INTERMACS) analysis of hospitalization, functional status, and mortality after mechanical circulatory support in adults with congenital heart disease. J Hear Lung Transplant. 2018.

\section{Figure Legends}

1. 3D-recontruction at ten years after implantation. 2. Chest X-ray after ten years from implantation. 

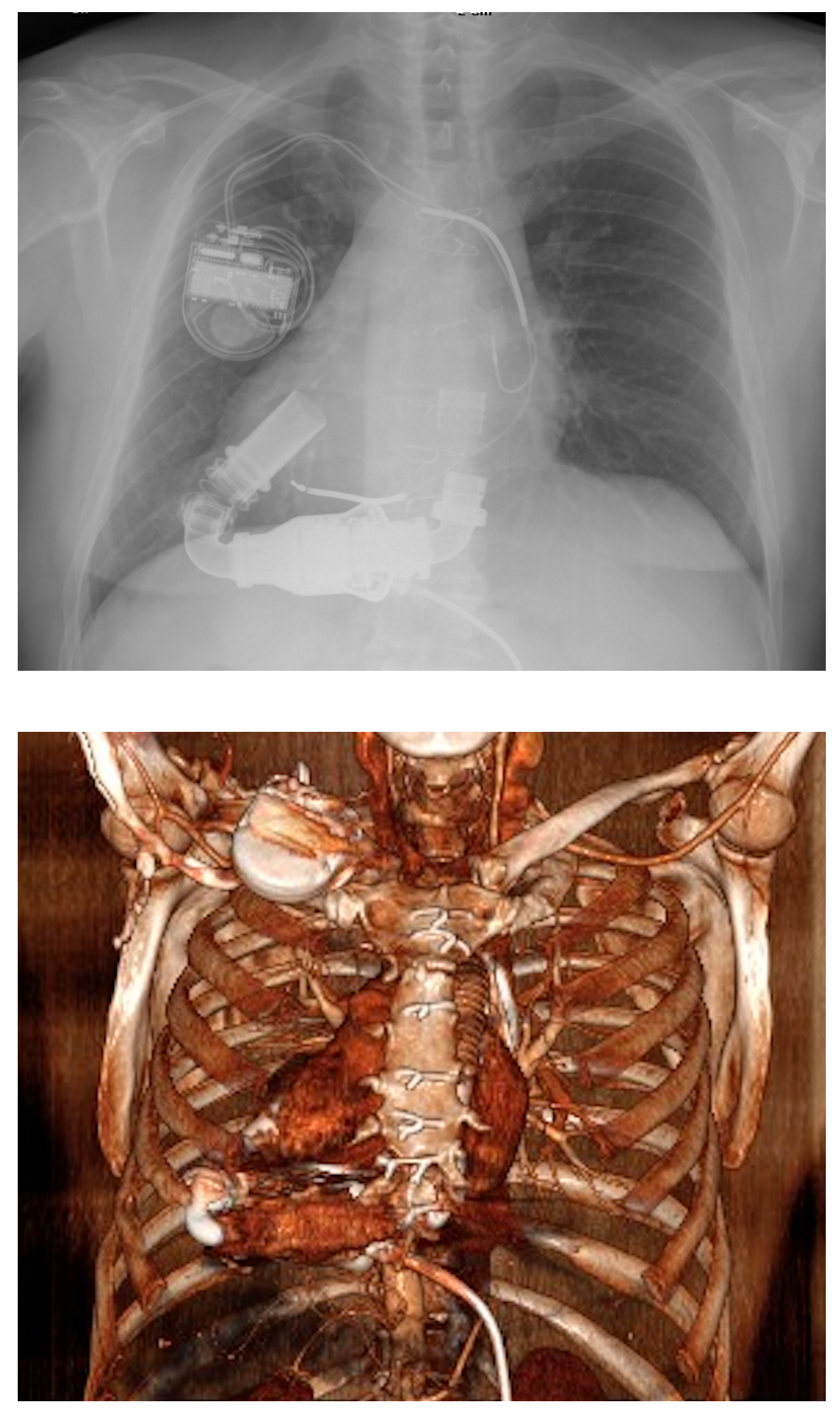